\title{
Phospho-Tau Antibody Delivery Using Virus-Like Nanoparticles as Carriers Enhances Spatial Memory Functions in Tauopathy Model Rats
}

\author{
Susheel Kumar Joseph ${ }^{1}$, Vishnuvardhan², C Sushmitha², Usha Natraj², Handanahal Subbarao Savithri², \\ Sarada Subramanian ${ }^{1 *}$ \\ ${ }^{1}$ Department of Neurochemistry, National Institute of Mental Health and Neurosciences, Bangalore, India \\ ${ }^{2}$ Department of Biochemistry, Indian Institute of Science, Bangalore, India

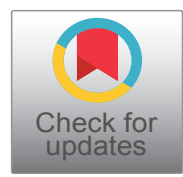

\begin{abstract}
Background: Anti-tau immunotherapies targeting phospho-epitopes have shown promising outcome in pre-clinical studies, although, with certain limitations in terms of mode of antibody delivery and concentration of antibodies required to obtain positive outcome.

Results \& methods: In this study, virus-like particles containing 180 binding sites for antibodies per particle was chosen as the antibody delivery tool. Antibodies against phospho-tau-peptide (sequence 50-71 AA) phosphorylated at S68, T69, and T71 were coated onto chimeric Sesbania mosaic virus-like nanoparticles containing B domain from $S$. aureus (SLB). SLB bound antibodies showed enhanced binding to tau protein as compared to unconjugated antibodies. Passive immunization studies were conducted in okadaic acid (OKA) induced tauopathy model rats. In Barnes maze task, SLB-Ab treated rats exhibited marked learning ability in comparison to OKA rats, with progressive decrease in the number of errors made and the time taken in reaching the escape hole.
\end{abstract}

Conclusions: These results suggest the merits of SLB-Ab in improving spatial memory as reflected by far lesser number of SLB-Ab injections required to observe the therapeutic effect.

\section{Keywords}

Antibody delivery, Okadaic acid, Phospho-tau, Spatial memory, Tauopathy, Virus-like nanoparticles

\section{Introduction}

Tauopathies are a class of neurodegenerative disorders [1] which include progressive supranuclear palsy, fronto temporal dementia, Alzheimer's disease (AD), etc. Pathologically tauopathies are characterized by accumulation of neurofibrillary tangles (NFT) inside the cell which are composed of abnormally hyperphosphorylated microtubule-associated protein tau (MAPT) or tau protein. Along with NFT and other pathological hallmark viz., insoluble amyloid $\beta$ plaques, $A D$ is a leading cause of dementia worldwide in the elderly population [2]. Tau proteins require phosphorylation for their binding to microtubule and to perform normal physiological functions such as microtubule stabilization and axonal transport [3]. However, in pathological conditions such as AD, tau phosphorylation is shown to be increased [4]. This hyperphosphorylated tau tends to aggregate and form paired helical filaments which subsequently form neurofibrillary tangles [5].

Hyperphosphorylation of tau protein is due to increased activity of tau kinases such as GSK3ß and cdk5 [6,7] and decreased activity of protein phosphatases including PP1 and
PP2A [8]. PP2A activity was shown to be reduced by approximately $50 \%$ in $A D$ brains [9]. It has been shown that specific serine-threonine residues are phosphorylated during pathological conditions such as AD that are not phosphorylated in normal physiological conditions [10]. Also, studies have shown that this hyperphosphorylated tau protein correlated well with cognitive deficits in AD cases [11]. Hence targeting hyperphosphorylated tau has been considered to be an effec-

*Corresponding author: Sarada Subramanian, Department of Neurochemistry, National Institute of Mental Health \& Neurosciences, Bangalore, 560 029, India, Tel: 91-80-26995165, Fax: 91-80-2656 4830, E-mail: sarada@nimhans.ac.in

Accepted: November 28, 2020

Published online: November 30, 2020

Citation: Joseph SK, Vishnuvardhan, Sushmitha C, et al. (2020) Phospho-Tau Antibody Delivery Using Virus-Like Nanoparticles as Carriers Enhances Spatial Memory Functions in Tauopathy Model Rats. Alzheimers Dis Dement 4(1):99-104 
Citation: Joseph SK, Vishnuvardhan, Sushmitha C, et al. (2020) Phospho-Tau Antibody Delivery Using Virus-Like Nanoparticles as Carriers Enhances Spatial Memory Functions in Tauopathy Model Rats. Alzheimers Dis Dement 4(1):99-104

tive therapeutic approach to treat AD.

Several approaches have been made to inhibit hyperphosphorylation of tau including inhibition of tau kinases [12], activation of tau phosphatases [13], clearance of tau aggregates by immunotherapy. Among these, immunotherapy targeting phospho-tau (hyperphosphorylated tau) has shown promising outcomes in improving cognitive functions in pre-clinical studies [14]. Earlier studies from our laboratory have shown that antibodies to phospho-tau peptide targeting phosphorylated residues in N-terminal part of tau (residues 50-71 and residues 111-137) improve spatial learning and memory in okadaic acid (OKA) induced tauopathy model rats with concomitant reduction in the phospho-tau levels in the hippocampal homogenates $[15,16]$. However, multiple injections of higher doses of antibodies were required for prolonged duration to see improvements in the memory functions indicating the need for enhanced antibody delivery systems. In this regard, plant virus based bionanoparticles as carriers are gaining momentum for their advantages in terms of biocompatibility and non-pathogenicity in humans. Further, these nanoparticle cages have the potential to provide effective delivery either by encapsulating the therapeutic cargo such as chemotherapeutic drugs, small inhibitory nucleic acids, peptides etc. or by tagging them on their surface such as antibodies with a much higher cargo/carrier ratio [17] as well as specific cell targeting. Encouraged with this, in the current study, the application of virus-like nanoparticles as antibody delivery tool was assessed. The therapeutic potential of phospho-tau antibodies delivered through virus-like nanoparticles in OKA induced tauopathy model rats and their effect in improving spatial memory was explored.

\section{Materials and Methods}

\section{Chemicals}

Okadaic acid, bovine serum albumin, Folin-ciocalteau's phenol reagent, goat anti-rabbit IgG fragment-horse radish peroxidase conjugate, 3,3',5,5'- tetramethyl benzidine, DMSO, protein-A-Sepharose were purchased from Sigma Aldrich, Bangalore, India. Anti-phospho-tau peptide serum used in the present study was raised earlier in rabbits and characterized [15]. Cloning, expression and purification of Sesbania mosaic virus (SeMV) chimeric virus like particles (SLB) containing SeMV coat protein with B domain from Staphylococcus aureus protein A fused at the $\mathrm{HI}$ loop region were described earlier [18]. All other reagents used were of analytical grade and purchased locally.

\section{Animals}

Five months old Sprague Dawley (SD) female rats weighing 210-250 g were procured from the Institute's Central Animal Research Facility. All the experiments involving the usage of animals were approved by Institute animal ethics committee (IACE No. AEC/68/434/N.C). The animals were housed in a controlled environment of light: dark (12 h:12 h) schedule, fed with pellet diet and water ad libitum.

\section{Preparation and characterization of SLB-Ab}

SLB was over expressed and purified from $E$. coli by meth- od described by [18]. Briefly, plasmid constructs containing SeMV capsid protein (C.P) or chimeric C.P containing SeMV capsid gene with the B-domain gene segment from $S$. aureus inserted at the HI loop region (SLB) were over expressed by inducing with IPTG. Purified virus like particles of SLB/SeMV C.P $(360 \mu \mathrm{g} / \mu \mathrm{L}$ or $0.16 \mu \mathrm{M})$ were incubated with antibodies to $\mathrm{P}$-tau peptide $(7.3 \mu \mathrm{M})$ overnight at $4{ }^{\circ} \mathrm{C}$ in the ratio of $1: 4$ (antibody to potentially available 180 antibody binding sites on the SLB). Then the solution was subjected to ultracentrifugation at 26000 rpm for 3 hours using Beckman Coulter SW32 rotor to separate unbound antibodies. The supernatant containing free antibodies were discarded and the pellet was suspended in $50 \mathrm{mM}$ Tris $\mathrm{HCl} \mathrm{pH} \mathrm{7.5.} \mathrm{Further,} \mathrm{this} \mathrm{solution} \mathrm{was}$ characterized by ELISA and used for immunotherapy studies. ELISA was performed to assess binding of SLB-Ab to tau protein. Briefly, $1 \mu \mathrm{g} /$ well in $0.1 \mathrm{M}$ phosphate buffered saline (PBS) of synthetic phospho-tau peptide (TPTEDGSEEPGSETSDAKpSpTPpT - residues 50-71 of full length human tau) or the recombinant full length human tau protein (441 amino acids long) was coated onto 96-well ELISA plate. The antigen coated wells were probed with $20 \mathrm{ng}$ of either SLB-Ab or unconjugated antibody were added. C.P ( $20 \mathrm{ng} /$ well) served as negative control. The antigen-antibody binding was quantitated by the addition of goat anti-rabbit IgG- horse radish peroxidase followed by the addition of tetramethyl benzidine as the substrate. After arresting the reaction, O.D was measured at 450 $\mathrm{nm}$ in an ELISA reader.

\section{Passive immunization studies}

Using the OKA-induced tauopathy model rats [15], passive immunization studies were conducted with SLB-Ab and the study design is described in Figure 1. On day 1 of the experiment, rats were administered with a single dose of $500 \mathrm{ng}$ of okadaic acid in 10 microlitres of $10 \%$ DMSO through intranasal route, administered as $5 \mu \mathrm{l}$ per nostril. On day 2 of the experimental procedure, rats have received first intraperitoneal injection of SLB-Ab conjugate. Self-assembled SeMV capsid protein as virus like particles devoid of B domain (C.P) served as negative control. Second injection of SLB-Ab or CP were administered after seven days of the first injection to respective group. The next day after the second injection, OKA rats were subjected to the Barnes circular maze test, as described by [19], with slight modifications. Briefly, animals were subjected to one day of habituation followed by three days of acquisition training which included 15 trials ( 3 trials/ day) and learning was assessed. Further, after four days of gap memory retention was assessed. The time taken for the rat to find and enter into the dark favorable escape box from open, bright and aversive environment on the maze (latency time) was recorded. Number of nose pokes into holes other than target hole containing the escape box (errors) was also recorded.

\section{Statistical analysis}

The data expressed as Mean \pm SEM. The data were analysed with GraphPad prism 8 (San Diego, CA, USA). Results of Barnes maze task were analysed by one way ANOVA followed by Tukeys multiples comparison post hoc test. $P$ values $<0.05$ were considered statistically significant. 


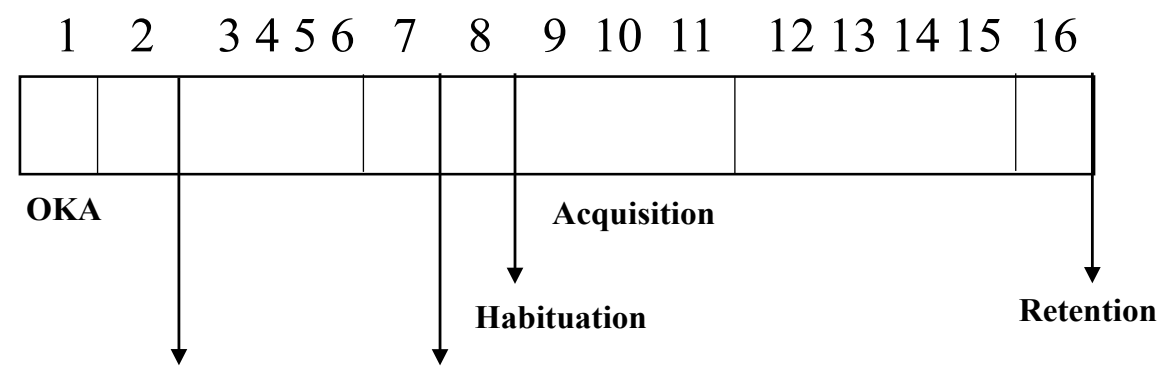

SLB-Ab/C.P

SLB-Ab/C.P

(1) C.P Group

(2) 1-dose SLB-Ab Group

(3) 2-dose SLB-Ab Group
C.P
C.P
C.P
SLB-Ab
SLB-Ab
SLB-Ab

Figure 1: Passive immunotherapy study design. The experiment was initiated by intranasal administration of okadaic acid (OKA; $2 \mu \mathrm{g} /$ Kg body weight) on day 1 of the experiment to 5-month-old SD rats. Details of Ab treatment to the different groups recruited in the study are indicated ( $n=6$ in each group). On day 2 and day 7, SLB-Ab complex (300 $\mu \mathrm{g} / 100 \mu \mathrm{l}$ saline) or CP (350 $\mu \mathrm{g} / 100 \mu \mathrm{l} \mathrm{saline)} \mathrm{were}$ injected through intraperitoneal route. On day 8 , the animals were habituated to Barnes maze. During days 9-11, the rats underwent training (acquisition phase). After a gap of 4 days, i.e., on day 16, retention studies were conducted.

\section{Results and Discussion}

Progressive accumulation and spread of hyperphosphorylated tau protein are the characteristic of tauopathies including AD. Increase in tau aggregates correlates well with cognitive deficits in AD cases [20]. Hence targeting hyperphosphorylated tau protein is an attractive therapeutic approach to $A D$. In line with this, therapeutic antibodies targeting specific phospho-peptides at N-terminal part [residues 25-30 [11], 5071 [15], 111-137 [16]] as well as C-terminal half (residues 199404) [21] of pathological tau have shown promising outcome in pre-clinical studies in improving cognitive functions. Also, multiple antibodies to phospho-tau are under various stages of clinical trials [11]. However, there are still limitations to this approach in terms of antibody delivery and concentration of antibodies required to obtain positive outcome. In the current study, genetically engineered SeMV coat protein harbouring 58 amino acid long B domain (SLB) was chosen as antibody carrier because itself assembles to form icosahedral particles with an average diameter of $37 \mathrm{~nm}$ [18]. These SLB particles are genome free, non-infectious capsids which retain the ability to bind antibodies after the assembly. More importantly, SLB have been shown to be the carriers for delivery of antibodies, targeting cellular as well as surface exposed antigens with long half-life of $8 \mathrm{~h}$ [18].

In the present study, antibodies raised against a synthetic peptide specific to the phospho epitope consisting of a cluster of three phosphorylated residues (S68, T69 and T71) within the amino acid sequence 50-71 of tau protein [15] were coated onto SLB (SLB-Ab) and used for immunotherapy experiments. To study the effect of immunotherapy, OKA induced tauopathy model was employed. OKA was administered intranasally to avoid higher doses, systemic side effects and to bypass blood-brain barrier [22]. OKA is a PP2A inhibitor, decreased activity of PP2A is observed in AD brain resulting in decreased dephosphorylation of tau proteins leading to $A D$ like hyperphosphorylation and aggregation of tau proteins in

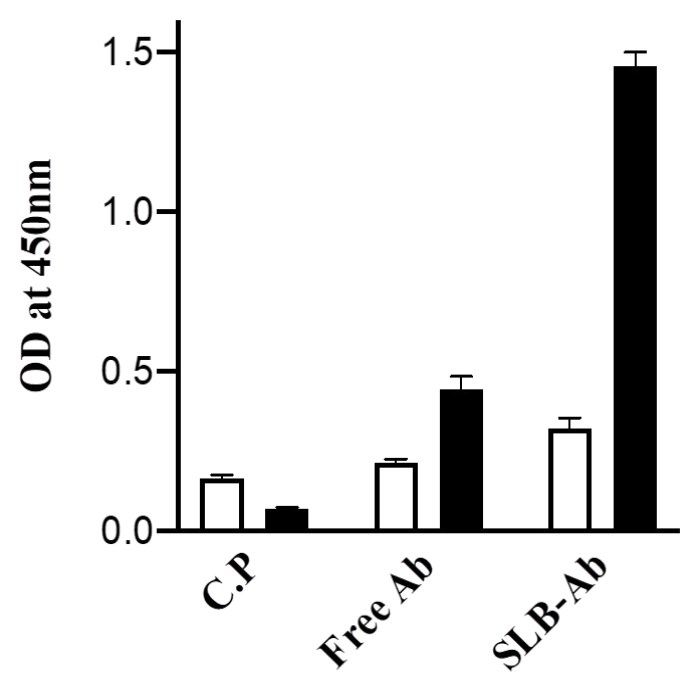

Figure 2: Comparison of the recognition of antibodies to phospho-tau peptide and full length tau protein in ELISA. Shown are the extent of binding to $1 \mu \mathrm{g} /$ well of phospho-Tau peptide ( $\square$ ) by C.P (devoid of any antibody, as a negative control) (0.152 \pm 0.022 ); free (unconjugated) antibodies to phosho-tau peptide $(0.207 \pm 0.018)$ and SLB-Ab complex $(0.315 \pm 0.039)$ or $1 \mu \mathrm{g} /$ well of full length tau protein $(\boldsymbol{\nabla})$ by C.P $(0.054 \pm 0.01)$; free (unconjugated) antibodies to phosho-tau peptide (0.446 \pm $0.043)$ and SLB-Ab complex $(1.478 \pm 0.05)$. Values presented as mean \pm SEM. The experiment was repeated thrice.

the form of NFTs [23] which is one of the pathological hall marks of $A D$ including synaptic and neuronal degeneration [24]. OKA also induces loss of neurons in hippocampus, a primary brain region involved in learning and memory functions resulting in impairment in spatial memory and learning [6].

\section{In vitro characterization of SLB-Ab particles}

The SLB-Ab complex prepared as described in the Methods section was tested for the ability to bind to full length 
Citation: Joseph SK, Vishnuvardhan, Sushmitha C, et al. (2020) Phospho-Tau Antibody Delivery Using Virus-Like Nanoparticles as Carriers Enhances Spatial Memory Functions in Tauopathy Model Rats. Alzheimers Dis Dement 4(1):99-104

recombinant tau protein or phospho-tau peptide coated to ELISA plates at $1 \mu \mathrm{g} /$ well. The ELISA results represented in Figure 2 demonstrate that SLB-Ab complex bind to the cognate tau peptide only marginally better when compared to the antibody alone and as expected, the virus-like particles (C.P) without the B-domain did not form the complex with the antibodies and hence showed negligible binding to the peptide. Of importance is the fact that, SLB-Ab complex showed enhanced binding with full-length tau protein compared to tau peptide, with the intensity of binding higher than the binding of the unconjugated antibodies to the full length protein. It may be noted that the same protein concentration (20 ng) of the free $A b$ as well as the SLB-Ab complex was used and therefore, the molar concentration of the Ab in SLB-Ab complex would be much lower than that of $A b$. In spite of this, the complex bound to tau protein much more efficiently (Figure 2).These results suggest that, the antibodies bind to the native protein antigen to a greater extent, when presented in the SLB-bound form vis-à-vis unconjugated state, thus implying improved therapeutic potential in vivo.

\section{Passive immunotherapy using SLB-Ab conjugate in tauopathy rats}

Using the OKA-induced tauopathy model rats [15], passive immunization studies were conducted with SLB-Ab as per the study design described in Figure $1 . A D$ is associated with a progressive deterioration of cognitive functions, including spatial disorientation and episodic memory failures in the affected human subjects [25] and animal models [26]. In order to assess the $A D$ associated spatial memory functions, the next day after the second injection, OKA rats were subjected to the Barnes circular maze test, a hippocampus-dependent cognitive task that requires spatial reference memory [27].

On the first day of the training session, all the rats from different groups committed more errors during exploration of the maze and, by day 3, the OKA rats treated with two doses of SLB-Ab showed significant improvement in spatial learning (Figure $3 \mathrm{~A}$ and Figure $3 \mathrm{~B}$ ) compared to rats treated with C.P particles and with single dose of SLB-Ab particles. Significant reduction in the number of errors during acquisition phase was appreciated starting from Day 2 in the case of OKA rats treated with two doses of SLB-Ab and the improved performance continued to Day 3 as well (Figure 3B).

The retention test was conducted after 4 days of acquisition to assess the consolidation of the memory. When the time taken to find the escape box and the number of errors made were recorded, it was observed that animals which have received two doses of SLB-Ab exhibited a significant reduction in the latency (Figure $4 \mathrm{~A}$ ) in accomplishing the task of finding the escape hole with negligible number of errors when compared to the other two groups (Figure 4B).

In our earlier study, a total of $6 \mathrm{mg}$ of the free $A b$, administered as $1 \mathrm{mg}$ / injection on 6 alternate days over a period of 12 days was required to elicit improvement in Barnes maze task by OKA rats [15]. It is very encouraging to note from the current study that, $\mathrm{Ab}$ administered as 2 injections of 300 $\mu \mathrm{g}$ of SLB-Ab complex each time achieved marked improvement in spatial memory functions at several-fold lower concentration of the antibodies. More importantly, this could be achieved with far lesser number of injections. In terms of the mechanism of action of these $A b$, it is possible that improvement in learning and memory functions may be due to SLB-Ab mediated clearance of intracellular and/or extracellular tau aggregates or pathological tau monomers by one or more following mechanisms. (i) Anti-P-tau antibodies can bind to extracellular tau aggregates and inhibit seeding and trans-cellular propagation of tau pathology [28]. (ii) Extracellular tau protein and anti-tau antibody complex can be cleared by mi-
(A)

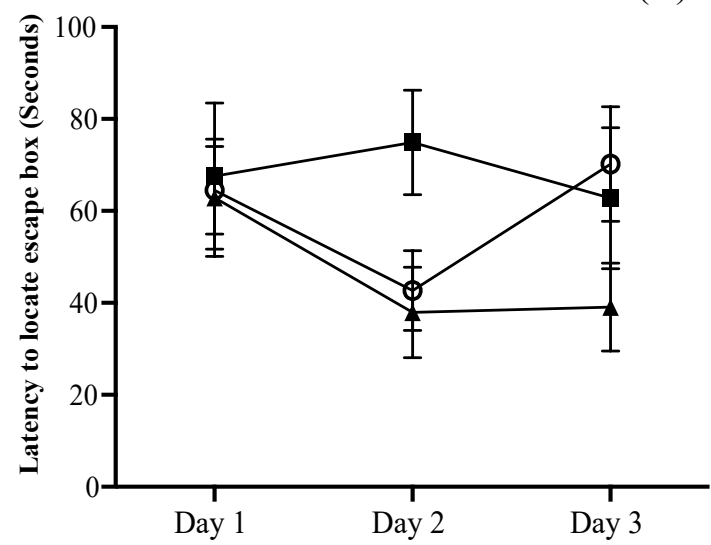

(B)



Figure 3: Performance of the experimental rats during the acquisition phase in Barnes maze task ( $n=6$ in each group). A) Latency to locate and enter into the escape box was measured during different days of acquisition phase. Although the latency was reduced on Day 3 in the OKA rats treated with two doses of SLB-Ab ( $\mathbf{\Delta} ; 38 \pm 10 \mathrm{sec}$ ), it was statistically insignificant, when compared to the animals which have received one dose of SLB-Ab ( $\mathbf{\square} ; 62 \pm 14 \mathrm{sec}$ ) or CP alone (0; $70 \pm 12 \mathrm{sec}$ ); B) Number of errors made during different days of acquisition phase of Barnes maze task was measured to assess the progress of learning. The OKA rats treated with two doses of SLB$\mathrm{Ab}$ made significantly lesser number of errors $(1.2 \pm 0.44)$ when compared to one dose of SLB-Ab group (4.1 \pm 0.9$)$ or CP alone group $\left(3.4 \pm 0.4 ;{ }^{* *} \mathrm{P}<0.01\right)$. Further, the data was significant $\left({ }^{* *} \mathrm{P}<0.01\right)$ when compared to the errors recorded on Day 1 of acquisition. Data presented as mean \pm SEM. 



Figure 4: Performance of the experimental rats during the retention phase in Barnes maze task ( $n=6$ in each group). A) Latency to locate and enter into the escape box was measured. OKA rats treated with two doses of SLB-Ab showed significantly lesser time (41 \pm 9.6 sec) to locate and enter into the escape box compared to one dose of SLB-Ab group ( $77 \pm 8.9 \mathrm{sec})$ or CP alone group $\left(80 \pm 11.6 \mathrm{sec}\right.$; $\left.{ }^{* *} \mathrm{P}<0.01\right)$; B) OKA rats treated with two doses of SLB-Ab made significantly lesser errors (0.4 \pm 0.1$)$ compared one dose of SLB-Ab group (6.4 \pm 0.15 ) or $\mathrm{CP}$ alone group $\left(5 \pm 0.2,{ }^{* * * *} \mathrm{P}<0.0001\right)$. Data presented as mean $\pm \mathrm{SEM}$.

croglial phagocytosis [29] (iii) SLB particles have been shown to efficiently deliver antibodies intracellularly [18], antibodies entered inside the cell can bind to and clear tau aggregates by endosomal-lysosomal pathway [29]. It has been shown that $50 \%$ of antibodies efflux across BBB within 48 minutes of injection and it occurs by Fc-receptor mediated process [30]. Since the Fc region of the antibodies bind to SLB particle, it is possible that Fc-receptor mediated efflux of antibodies across BBB is prevented thus enhancing the availability of antibodies in the brain. Further experiments are warranted to assess the duration of the efficacy of therapeutic antibodies and their mechanism of action.

\section{Acknowledgements}

Financial assistance to SS from Council of Scientific \& Industrial Research, New Delhi [Grant no.37 (1607) 13/ EMRII] and Indian Council of Medical Research, New Delhi [Grant no.5/4-5/181/2016-NCD-I] are gratefully acknowledged. HSS thanks National Academy of Science India (NASI) for the senior scientist position. We thank Ambily Abraham for the SLB and C.P constructs used in this study. We are thankful to Prof. Srinivas Bharath, Department of Clinical Pharmacology \& Neurotoxicology, National Institute of Mental Health \& Neurosciences, Bangalore for his critical inputs in preparation of the manuscript.

\section{References}

1. Lee VM, Goedert M, Trojanowski JQ (2001) Neurodegenerative tauopathies. Annu Rev Neurosci 24: 1121-1159.

2. Ferri CP, Prince M, Brayne $C$, et al. (2005) Alzheimer's disease international: Global prevalence of dementia: A Delphi consensus study. Lancet 366: 2112-2117.

3. Roy S, Zhang B, Lee VM, et al. (2005) Axonal transport defects: A common theme in neurodegenerative diseases. Acta Neuropathol 109: 5-13.

4. Buee L, Bussiere T, Buee-Scherrer V, et al. (2000) Tau protein isoforms phosphorylation and role in neurodegenerative disorders Brain Research. Brain Res Brain Res Rev 33: 95-130.

5. Lewis J, Dickson DW, Lin WL, et al. (2001) Enhanced neurofibrillary degeneration in transgenic mice expressing mutant tau and APP. Science 293: 1487-1491.

6. Hooper C, Killick R, Lovestone S (2008) The GSK3 hypothesis of Alzheimer's disease. J Neurochem 104: 1433-1439.

7. Liu SL, Wang C, Jiang T, et al. (2016) The role of Cdk5 in Alzheimer's disease. Mol Neurobiol 53: 4328-4342.

8. Liu F, Grundke-lqbal I, Iqbal K, et al. (2005) Contributions of protein phosphatases PP1 PP2A PP2B and PP5 to the regulation of tau phosphorylation. Eur J Neurosci 221: 942-1950.

9. Gong CX, Singh TJ, Grundke-lqbal I, et al. (1993) Phosphoprotein phosphatase activities in Alzheimer disease brain. J Neurochem 61: 921-927.

10. Simic G, Leko MB, Wray S, et al. (2016) Tau protein hyperphosphorylation and aggregation in Alzheimer's Disease and other tauopathies and possible neuroprotective strategies. Biomolecules 6: 6 .

11. Sigurdsson EM (2018) Tau immunotherapies for Alzheimer's disease and related tauopathies: Progress and potential pitfalls. J Alzheimers Dis 64: S555-S565.

12. Khalil H, Mitev V, Vlaykova T, et al. (2015) Discovery and development of Seliciclib how systems biology approaches can lead to 
Citation: Joseph SK, Vishnuvardhan, Sushmitha C, et al. (2020) Phospho-Tau Antibody Delivery Using Virus-Like Nanoparticles as Carriers Enhances Spatial Memory Functions in Tauopathy Model Rats. Alzheimers Dis Dement 4(1):99-104

better drug performance. Journal of Biotechnology 202: 40-49.

13. Matsunaga S, Kishi T, Iwata N (2015) Memantine monotherapy for Alzheimer's Disease: A systematic review and meta-analysis. PLOS ONE 10: e0123289.

14. Asuni AA, Boutajangout A, Quartermain DandSigurdsson EM (2007) Immunotherapy targeting pathological tau conformers in a tangle mouse model reduces brain pathology with associated functional improvements. J Neurosci 27: 9115-9129.

15. Subramanian S, Savanur G, Madhavadas S (2017) Passive immunization targeting the $\mathrm{N}$-terminal region of phosphorylated tau (residues 68-71) improves spatial memory in okadaic acid induced tauopathy model rats. Biochem Biophys Res Commun 483: 585-589.

16. Subramanian S, Savanur G (2020) Antibodies directed to the phospho-tau peptide (residues 111-137) dissociate tau oligomers and reduce the spatial memory deficits in non-transgenic tauopathy model rats. Indian J Exp Biol 58: 355-359.

17. Rohovie MJ, Nagasawa M, Swartz JR (2017) Virus-like particles: Next-generation nanoparticles for targeted therapeutic delivery. Bioeng Transl Med 2: 43-57.

18. Abraham A, Natraj U, Karande AA, et al. (2016) Intracellular delivery of antibodies by chimeric Sesbania mosaic virus (SeMV) virus like particles. Sci Rep 6: 21803.

19. Madhavadas S, Subramaninan S, Kutty BM (2017) Environmental enrichment improved cognitive deficits more in peri-adolescent than in adult rats after postnatal monosodium glutamate treatment. Physiol Int 104: 271-290.

20. Congdon E, Sigurdsson EM (2018) Tau-targeting therapies for Alzheimer disease. Nat Rev Neurol 14: 399-415.

21. Schroeder SK, Joly-Amado A, Gordon MN, et al. (2016) Tau-di- rected immunotherapy: A promising strategy for treating Alzheimer's disease and other tauopathies. J Neuroimmune Pharmacol 11: 9-25.

22. Erdo F, Bors LA, Farkas D, et al. (2018) Evaluation of intranasal delivery route of drug administration for brain targeting. Brain Res Bull 143: 155-170.

23. Kamat KP, Rai S, Nath C (2013) Okadaic acid induced neurotoxicity: An emerging tool to study Alzheimer's pathology. Neurotoxicology 37: 163-172.

24. Natalia CB, Clara T, Fred VL (2012) Protein Tau: Prime cause of synaptic and neuronal degeneration in Alzheimer's disease. International Journal of Alzheimers Disease 2012: 251426.

25. Finkel SI (2003) Behavioural and psychologic symptoms of dementia. Clinics in Geriatric Medicine 19: 799-824.

26. Reiserer RS, Harrison FE, Syverud DC, et al. (2007) Impaired spatial learning in the APPSwe+PSEN1DeltaE9 bigenic mouse model of Alzheimer's disease. Genes Brain Behav 6: 54-65.

27. Bach ME, Hawkins RD, Osman M, et al. (1995) Impairment of spatial but not contextual memory in CaMKII mutant mice with a selective loss of hippocampal LTP in the range of the theta frequency. Cell 81: 905-915.

28. Yanamandra K, Kfoury N, Jiang H, et al. (2013) Anti-tau antibodies that block tau aggregates seeding in vitro markedly decrease pathology and improve cognition in vivo. Neuron 80: 402-414.

29. Sigurdsson EM (2008) Immunotherapy targeting pathological tau protein in Alzheimer's disease and related tauopathies. J Alzheimers Dis15: 157-168.

30. Zhang Y, Pardridge WM (2001) Mediated efflux of IgG molecules from brain to blood across the blood-brain barrier. J Neuroimmunol 114: 168-172.

DOI: $10.36959 / 734 / 379$

Copyright: (C) 2020 Joseph SK, et al. This is an open-access article distributed under the terms of the Creative Commons Attribution License, which permits unrestricted use, distribution, and reproduction in any medium, provided the original author and source are credited. 